\title{
Logistic Regression Model Using Scheimpflug-Placido Cornea Topographer Parameters to Diagnose Keratoconus
}

\author{
Emre Altinkurt $\mathbb{D}^{1},{ }^{1}$ Ozkan Avci $\mathbb{D}^{1},{ }^{1}$ Orkun Muftuoglu $\mathbb{D}^{\mathbb{D}},{ }^{2}$ Adem Ugurlu $\mathbb{D}^{\mathrm{D}},{ }^{3}$ \\ Zafer Cebeci $\mathbb{D}^{1},{ }^{1}$ and Kemal Turgay Ozbilen $\mathbb{D}^{1}$ \\ ${ }^{1}$ Istanbul University, Istanbul Faculty of Medicine, Department of Ophthalmology, Fatih/Capa, Istanbul 34093, Turkey \\ ${ }^{2}$ FEBO, Professor of Ophthalmology, Koc University, Faculty of Medicine Department of Ophthalmology, \\ Zeytinburnu/Istanbul 34010, Turkey \\ ${ }^{3}$ Erzincan University, Faculty of Medicine, Department of Ophthalmology, Fatih, Erzincan 24100, Turkey
}

Correspondence should be addressed to Emre Altinkurt; altinkurtemre@gmail.com

Received 13 February 2021; Revised 11 April 2021; Accepted 10 May 2021; Published 19 May 2021

Academic Editor: Vincent M. Borderie

Copyright $(92021$ Emre Altinkurt et al. This is an open access article distributed under the Creative Commons Attribution License, which permits unrestricted use, distribution, and reproduction in any medium, provided the original work is properly cited.

\begin{abstract}
Purpose. Diagnose keratoconus by establishing an effective logistic regression model from the data obtained with a ScheimpflugPlacido cornea topographer. Methods. Topographical parameters of 125 eyes of 70 patients diagnosed with keratoconus by clinical or topographical findings were compared with 120 eyes of 63 patients who were defined as keratorefractive surgery candidates. The receiver operating character (ROC) curve analysis was performed to determine the diagnostic ability of the topographic parameters. The data set of parameters with an AUROC (area under the ROC curve) value greater than 0.9 was analyzed with logistic regression analysis (LRA) to determine the most predictive model that could diagnose keratoconus. A logit formula of the model was built, and the logit values of every eye in the study were calculated according to this formula. Then, an ROC analysis of the logit values was done. Results. Baiocchi Calossi Versaci front index $\left(\mathrm{BCV}_{\mathrm{f}}\right)$ had the highest AUROC value $(0.976)$ in the study. The LRA model, which had the highest prediction ability, had $97.5 \%$ accuracy, $96.8 \%$ sensitivity, and $99.2 \%$ specificity. The most significant parameters were found to be $\mathrm{BCV}_{\mathrm{f}}(p=0.001), \mathrm{BCV}_{\mathrm{b}}$ (Baiocchi Calossi Versaci back) $(p=0.002)$, posterior $\mathrm{rf}$ (apical radius of the flattest meridian of the aspherotoric surface in $4.5 \mathrm{~mm}$ diameter of the cornea) $(p=0.005)$, central corneal thickness $(p=0.072)$, and minimum corneal thickness $(p=0.494)$. Conclusions. The LRA model can distinguish keratoconus corneas from normal ones with high accuracy without the need for complex computer algorithms.
\end{abstract}

\section{Introduction}

Keratoconus is an ectatic disorder that is characterized by progressive stromal thinning and protrusion of the cornea with irregular astigmatism [1]. Ectasia progression after refractive surgery in patients with keratoconus has been reported in previous studies [2]. The prevalence of keratoconus is higher among refractive surgery candidates compared to the general population [3], and operating on a cornea with keratoconus can cause corneal ectasia after refractive surgery [4]. Topography systems are very useful in the diagnosis of keratoconus. Still, an exact diagnosis is difficult because threshold criteria remain to be defined. Moreover, examining each parameter in the topography device one by one takes time. This study aimed to gauge the most useful parameters of the Sirius Scheimpflug-Placido topographer in determining keratoconus eyes from normal eyes and to find an accurate logistic regression model for diagnosing keratoconus with these parameters.

\section{Patients and Methods}

The study was conducted in agreement with the ethical standards established in the Declaration of Helsinki and endorsed by the local clinical research ethics committee. Informed consent was obtained from all patients. The study consisted of 120 eyes from 63 patients who had undergone keratorefractive surgery (normal group) and 125 eyes from 
70 patients who were diagnosed with keratoconus (keratoconus group) between November 2012 and May 2015. The parameters provided by the Scheimpflug-Placido topographer (Sirius $3 \mathrm{D}$ Rotating Scheimpflug Camera and Topography System, Costruzione Strumenti Oftalmici, Italy) were evaluated retrospectively. Excluded were patients who were pregnant or lactating and who had corneal scarring, dry eye, concomitant corneal or ocular disease, previous corneal collagen crosslinking, ocular surgery, or trauma. Subjects were divided into normal and keratoconus groups.

All patients in the keratoconus group were diagnosed as keratoconus on the basis of topographic signs. In this study, keratoconus is defined as any eye that has an abnormal localized steepening or an asymmetric bow tie pattern with or without skewed axes, combined with at least one of the following signs: central corneal thickness $<500 \mu \mathrm{m}$, oblique cylinder $>1.5$ diopter (D), steep keratometry $>47 \mathrm{D}$, or clinical keratoconus in the fellow eye $[5,6]$. Myopic laser refractive surgery patients who were assigned to the normal group had preoperative sphere $<-6.50 \mathrm{D}$ and cylinder $<-3.00 \mathrm{D}$ without an irregular corneal topographic pattern. No forme fruste keratoconus patients were included among refractive surgery candidates.

Corneal scans were done under scotopic conditions without pupillary dilation by the same experienced operator using the Sirius Scheimpflug-Placido topographer. Acceptable-quality maps with at least $9.0 \mathrm{~mm}$ of corneal coverage were used in the study. All wavefront aberrations were performed for a $6 \mathrm{~mm}$ diameter pupil. The following parameters were recorded and analyzed: simulated keratometry measurements consisting of flat (Sim-K1) and steep (Sim-K2) corneal power; corneal dioptric power in the flattest and steepest meridian for both corneal surfaces in the $3.0 \mathrm{~mm}$ central corneal zone (anterior $\emptyset=3 \mathrm{~mm} \mathrm{~K} 1$, anterior $\emptyset=3 \mathrm{~mm} \mathrm{~K} 2$, posterior $\varnothing=3 \mathrm{~mm} \mathrm{~K} 1$, posterior $\varnothing=3 \mathrm{~mm}$ $\mathrm{K} 2$ ) and $5.0 \mathrm{~mm}$ central corneal zone (anterior $\emptyset=5 \mathrm{~mm} \mathrm{~K}$, anterior $\emptyset=5 \mathrm{~mm} \mathrm{~K} 2$, posterior $\varnothing=5 \mathrm{~mm} \mathrm{~K} 1$, posterior $\emptyset=5 \mathrm{~mm} \mathrm{~K} 2$ ); minimum corneal thickness (TCT), keratometry of the steepest point $\left(\mathrm{K}_{\max }\right)$ on the anterior tangential map, central corneal thickness (CCT), distance from the corneal endothelium to the anterior surface of the lens (AD), distance of the thinnest point to the center of the cornea (TCTr), distance of Kmax to the center of the cornea (Apex $r$ ), and corneal volume in the $10 \mathrm{~mm}$ central corneal zone $(\mathrm{CV})$; symmetry index front $\left(\mathrm{SI}_{\mathrm{f}}\right)$ and back $\left(\mathrm{SI}_{\mathrm{b}}\right)$; Baiocchi Calossi Versaci index front $\left(\mathrm{BCV}_{\mathrm{f}}\right)$ and back $\left(\mathrm{BCV}_{\mathrm{b}}\right)$, keratoconus vertex index front $\left(\mathrm{KV}_{\mathrm{f}}\right)$ and back $\left(\mathrm{KV}_{\mathrm{b}}\right)$; apical radius of the flattest (anterior $\mathrm{rf}$, posterior $\mathrm{rf}$ ) and steepest meridian (anterior rs, posterior rs) of the aspherotoric surface in the $4.5 \mathrm{~mm}$ zone of the cornea front and back, mean asphericity in the $4.5 \mathrm{~mm}$ zone of the cornea front (anterior Q) and back (posterior Q), and root mean square values per unit area in the $4.5 \mathrm{~mm}$ zone of the cornea front (anterior RMS/A) and back (posterior RMS/A); total wavefront error (OPD), higher-order aberrations (HOAs), astigmatism $Z(2, \pm 2)$, coma aberration $Z(3, \pm 1)$, spherical aberration $Z(4,0)$, and residual HOAs (noncoma, nonspherical) for total, anterior, and posterior surfaces of cornea; and Kmax/TCT and $\mathrm{Kmax}^{2}$ /TCT values.
All data were analyzed with the Statistical Package for the Social Sciences (SPSS) software (version 21.0, SPSS, Inc.). The Kolmogorov-Smirnov method was used to test the variables for normal distribution. Categorical variables were analyzed using the chi-square test. In the analysis of the difference between the two groups regarding quantitative variables, the normally distributed variables were tested using independent samples $t$-test, and the not normally distributed variables were tested with the Mann-Whitney $U$ test. Receiver operating characteristic (ROC) curves were used to evaluate the accuracy of the parameters. The area under the curve (AUROC), sensitivity, specificity, and cut-off values that matched the maximum AUROC were calculated and compared between the two groups. [7] The diagnostic power of the parameters in the study was defined according to the AUROC values as excellent (0.90-1.00), good (0.80-0.89), fair (0.70-0.79), poor (0.60-0.69), and very poor (0.50-0.59). [8] The data set of the parameters with AUROC values greater than 0.9 was analyzed with logistic regression analysis (LRA) to determine the simplest model that could diagnose keratoconus.

The predictive capability of the eight models was investigated using LRA: Model 1 was a cluster of anterior $\varnothing=3 \mathrm{~mm} \mathrm{~K} 2$, posterior $\varnothing=3 \mathrm{~mm} \mathrm{~K} 2, \mathrm{~K}_{\max }$, CCT, and TCT. Model 2 was a cluster of anterior shape parameters (anterior RMS/A, anterior rs, anterior rf, and anterior Q). Model 3 was a cluster of posterior shape parameters (posterior RMS/A, posterior rs, posterior rf, and posterior Q). Model 4 was an addition of Model 2 and Model 3. Model 5 consisted of anterior and posterior keratoconus indices $\left(\mathrm{BCV}_{\mathrm{f}}, \mathrm{BCV}_{\mathrm{b}}\right.$, $\mathrm{KV}_{\mathrm{f}}, \mathrm{KV}_{\mathrm{b}}, \mathrm{SI}_{\mathrm{f}}$, and $\mathrm{SI}_{\mathrm{b}}$ ). Model 6 was a cluster of all corneal aberrations.

The significant parameters in these models were selected and gathered in different combinations with the other parameters in the study to find Model 7 and Model 8, the most predictive models. The logit formula of Model 8 was built. Then, the logit value of every eye in the study was calculated according to the logit formula of Model 8. ROC analysis of the logit values was performed to find the cut-off value of the logit formula. The results were evaluated according to a confidence interval of $95 \%$, and a $p$ value $<0.05$ was considered statistically significant.

\section{Results}

There was no statistically significant difference between the keratoconus (mean age $31.03 \pm 9.62$ years) and normal groups regarding age (mean age $31.03 \pm 8.05$ years) distribution $(p>0.05)$. All topographic parameters of the two groups were statistically significantly different (Tables 1 and 2 ). The ROC curve analysis of the topographic parameters with AUROC values less than 0.9 is in Table 3 and that with AUROC values greater than 0.9 is in Table 4. The AUROC values of the anterior aberrations were greater than the AUROC values of total aberrations; the AUROC values of the anterior keratoconus indices were greater than the AUROC values of the posterior keratoconus indices, and the AUROC values of the keratoconus indices were greater than 
TABLE 1: The mean values of summary indices, keratoconus indices, specially calculated indices, keratometry readings, and keratoconus indices in keratoconus and normal groups.

\begin{tabular}{|c|c|c|c|}
\hline Parameter & Keratoconus mean \pm SD (range) & Normal mean \pm SD (range) & $p$ value* \\
\hline $\begin{array}{l}\text { Summary indices } \\
\text { TCT }(\mu \mathrm{m}) \\
\text { TCT } r(\mathrm{~mm}) \\
\text { CCT }(\mu \mathrm{m}) \\
K_{\max }(\mathrm{D}) \\
\text { Apex } r(\mathrm{~mm}) \\
\text { CV }\left(\mathrm{mm}^{3}\right) \\
\text { AD }(\mathrm{mm})\end{array}$ & $\begin{array}{c}445.08 \pm 49.19(212,542) \\
0.76 \pm 0.44(0.20,3) \\
459.5 \pm 49.95(243,557) \\
54.12 \pm 5.71(44.50,68.41) \\
1.31 \pm 0.79(0.20,3.40) \\
54.03 \pm 3.05(45.6,62.4) \\
3.3 \pm 0.32(2.57,4.24)\end{array}$ & $\begin{array}{c}540.28 \pm 33.12(465,607) \\
0.63 \pm 0.24(0.13,2) \\
543.59 \pm 31.98(471,611) \\
45.70 \pm 2.33(41.98,57.07) \\
1.67 \pm 0.97(0.20,3.40) \\
58.03 \pm 3.22(49.4,66.3) \\
3.13 \pm 0.34(2.4,3.95)\end{array}$ & $\begin{array}{l}0.01> \\
0.012 \\
0.01> \\
0.01> \\
0.01> \\
0.01> \\
0.01>\end{array}$ \\
\hline $\begin{array}{l}\text { Specially calculated indices } \\
\mathrm{Kmax} / \mathrm{TCT}(\mathrm{D} / \mu \mathrm{m}) \\
\mathrm{Kmax}^{2} / \mathrm{TCT}\left(\mathrm{D}^{2} / \mu \mathrm{m}\right)\end{array}$ & $\begin{array}{c}0.26 \pm 1.60(0.08,18.1) \\
0.049 \pm 0.54(0.0001,6.09)\end{array}$ & $\begin{array}{c}0.08 \pm 0.007(0.07,0.12) \\
0.0001 \pm 0.00002(0.0001,0.0002)\end{array}$ & $\begin{array}{l}0.01> \\
0.01>\end{array}$ \\
\hline $\begin{array}{l}\text { Keratometry readings } \\
\text { Sim-K1 (D) } \\
\text { Sim-K2 (D) } \\
\text { Anterior } \emptyset=3 \mathrm{~mm} \mathrm{K1} \text { (D) } \\
\text { Anterior } \emptyset=3 \mathrm{~mm} \mathrm{~K} 2 \text { (D) } \\
\text { Anterior } \emptyset=5 \mathrm{~mm} \mathrm{K1} \text { (D) } \\
\text { Anterior } \emptyset=5 \mathrm{~mm} \mathrm{K2} \text { (D) } \\
\text { Posterior } \emptyset=3 \mathrm{~mm} \mathrm{~K} 1 \text { (D) } \\
\text { Posterior } \emptyset=3 \mathrm{~mm} \mathrm{K2} \text { (D) } \\
\text { Posterior } \emptyset=5 \mathrm{~mm} \mathrm{K1} \text { (D) } \\
\text { Posterior } \varnothing=5 \mathrm{~mm} \mathrm{K2} \text { (D) }\end{array}$ & $\begin{array}{c}45.01 \pm 3.14(40.11,57.77) \\
48.19 \pm 4.02(40.92,62.46) \\
45.46 \pm 5.27(41.01,67.90) \\
49.72 \pm 3.43(40.82,60.03) \\
45.29 \pm 4.5(31.61,73.12) \\
49.06 \pm 5.96(40.96,86.01) \\
-6.37 \pm 1.5(-9.89,-0.26) \\
-7.96 \pm 1.69(-15.4,-1.02) \\
-6.26 \pm 1.25(-8.91,-0.44) \\
-7.38 \pm 1.48(-15.88,1.97)\end{array}$ & $\begin{array}{c}42.61 \pm 1.36(39.45,46.28) \\
43.94 \pm 1.53(41.15,49.46) \\
42.56 \pm 1.35(39.65,46.33) \\
44.06 \pm 1.57(41,49.99) \\
42.56 \pm 1.33(39.55,46.3) \\
43.95 \pm 1.54(41.06,49.58) \\
-5.88 \pm 0.36(-6.36,-2.57) \\
-6.37 \pm 0.39(-9.87,-5.82) \\
-5.9 \pm 0.31(-6.36,-3.36) \\
-6.35 \pm 0.27(-8.06,-5.82)\end{array}$ & $\begin{array}{l}0.01> \\
0.01> \\
0.01> \\
0.01> \\
0.01> \\
0.01> \\
0.01> \\
0.01> \\
0.01> \\
0.01>\end{array}$ \\
\hline $\begin{array}{l}\text { Keratoconus indices } \\
\mathrm{SI}_{\mathrm{f}}(\mathrm{D}) \\
\mathrm{KV}_{\mathrm{f}}(\mu \mathrm{m}) \\
\mathrm{BVC}_{\mathrm{f}}(\mathrm{D}) \\
\mathrm{SI}_{\mathrm{b}}(\mathrm{D}) \\
\mathrm{KV}_{\mathrm{b}}(\mu \mathrm{m}) \\
\mathrm{BCV}_{\mathrm{b}}(\mathrm{D})\end{array}$ & $\begin{array}{c}4.96 \pm 3.68(-7.36,14.82) \\
28.07 \pm 17.91(4,102) \\
2.74 \pm 1.95(0,11.81) \\
1.29 \pm 0.93(-1.77,4.46) \\
65.10 \pm 40.18(9,264) \\
2.74 \pm 1.90(0,9.83)\end{array}$ & $\begin{array}{c}-0.08 \pm 0.56(-1.43,2.35) \\
4.58 \pm 3.65(2,37) \\
0.13 \pm 0.19(0,1.01) \\
0.09 \pm 1.37(-0.77,15) \\
13.35 \pm 9.88(0,111) \\
0.08 \pm 0.15(0,0.80)\end{array}$ & $\begin{array}{l}0.01> \\
0.01> \\
0.01> \\
0.01> \\
0.01> \\
0.01>\end{array}$ \\
\hline
\end{tabular}

* Mann-Whitney $U$ test. $\mathrm{SD}=$ standard deviation; TCT = minimum corneal thickness; TCT $r=$ distance of the thinnest point to the center of the cornea; $\mathrm{CCT}=$ central corneal thickness; $K_{\max }=$ keratometry of the steepest point recorded from anterior tangential map; Apex $r=$ distance of $\mathrm{K}_{\max }$ to the center of the cornea; $\mathrm{CV}=$ corneal volume in $10 \mathrm{~mm}$ central corneal zone; $\mathrm{AD}=$ distance from the corneal endothelium to the anterior surface of the lens; Sim$\mathrm{K} 1=$ simulated flat keratometry; Sim-K2=simulated steep keratometry; $\mathrm{K} 1=$ flat keratometry; $\mathrm{K} 2=$ steep keratometry; $\emptyset=$ central corneal zone; $\mathrm{SI}_{\mathrm{f}}=$ symmetry index front; $\mathrm{KV}_{\mathrm{f}}=$ keratoconus vertex front index; $\mathrm{BCV}_{\mathrm{f}}=$ Baiocchi Calossi Versaci front index; $\mathrm{SI}_{\mathrm{b}}=$ symmetry index back; $\mathrm{KV}_{\mathrm{b}}=$ keratoconus vertex back index; $\mathrm{BCV}_{\mathrm{b}}=$ Baiocchi Calossi Versaci back index.

TABLE 2: The mean values of shape indices and aberrations in keratoconus and normal groups.

\begin{tabular}{|c|c|c|c|}
\hline Parameter & Keratoconus mean \pm SD (range) & Normal mean \pm SD (range) & $p$ value* \\
\hline \multicolumn{4}{|l|}{ Shape indices } \\
\hline Anterior rf (D) & $46.83 \pm 6.24(30.63,81.45)$ & $42.59 \pm 1.36(39.63,46.20)$ & $0.01>$ \\
\hline Anterior rs (D) & $51.15 \pm 8.60(40.86,113.3)$ & $43.95 \pm 1.53(41.03,48.91)$ & $0.01>$ \\
\hline Anterior Q & $0.01 \pm 1.17(-5.45,3.14)$ & $0.84 \pm 0.36(-0.22,1.47)$ & $0.01>$ \\
\hline Anterior RMS/A $\left(\mu \mathrm{m} / \mathrm{mm}^{2}\right)$ & $0.20 \pm 0.14(0.01,1.17)$ & $0.02 \pm 0.02(0.01,0.24)$ & $0.01>$ \\
\hline Posterior rf (D) & $-7.17 \pm 1.46(-12.76,-3.34)$ & $-5.93 \pm 0.22(-6.38,-4.73)$ & $0.01>$ \\
\hline Posterior rs (D) & $-7.69 \pm 5.32(-15.85,48.20)$ & $-6.06 \pm 1.95(-6.87,11)$ & $0.01>$ \\
\hline Posterior $(\mathrm{p})$ & $-0.36 \pm 1.60(-3.47,7.19)$ & $0.80 \pm 0.55(-0.58,4.33)$ & $0.01>$ \\
\hline Posterior RMS/A $\left(\mu \mathrm{m} / \mathrm{mm}^{2}\right)$ & $0.63 \pm 0.63(0.06,5.69)$ & $0.10 \pm 0.06(0.03,0.70)$ & $0.01>$ \\
\hline \multicolumn{4}{|l|}{ Aberrations } \\
\hline \multicolumn{4}{|l|}{ Total } \\
\hline $\mathrm{OPD}(\mu \mathrm{m})$ & $2.94 \pm 2.43(0.32,14.44)$ & $0.95 \pm 0.85(0.25,6.29)$ & $0.01>$ \\
\hline HOAs $(\mu \mathrm{m})$ & $1.57 \pm 1.35(0.19,10.18)$ & $0.37 \pm 0.42(0.15,4.64)$ & $0.01>$ \\
\hline Astigmatism Z $(2, \pm 2)(\mu \mathrm{m})$ & $2.37 \pm 2.15(0.02,13.37)$ & $0.83 \pm 0.78(0.02,4.25)$ & $0.01>$ \\
\hline Coma Z $(3, \pm 1)(\mu \mathrm{m})$ & $1.10 \pm 0.80(0.08,5.51)$ & $0.17 \pm 0.17(0.01,1.82)$ & $0.01>$ \\
\hline Spheric Z $(4,0)(\mu \mathrm{m})$ & $0.26 \pm 0.34(0.01,2.97)$ & $0.16 \pm 0.18(0.02,1.74)$ & 0.042 \\
\hline Residual HOAs $(\mu \mathrm{m})$ & $0.89 \pm 0.95(0.13,6.50)$ & $0.27 \pm 0.38(0.06,4.03)$ & $0.01>$ \\
\hline
\end{tabular}


TABLE 2: Continued.

\begin{tabular}{|c|c|c|c|}
\hline Parameter & Keratoconus mean \pm SD (range) & Normal mean \pm SD (range) & $p$ value* \\
\hline \multicolumn{4}{|l|}{ Anterior } \\
\hline $\mathrm{OPD}(\mu \mathrm{m})$ & $2.84 \pm 1.68(0.37,9.97)$ & $0.96 \pm 0.72(0.20,3.73)$ & $0.01>$ \\
\hline HOAs $(\mu \mathrm{m})$ & $1.62 \pm 1.09(0.23,8.59)$ & $0.27 \pm 0.11(0.14,1.05)$ & $0.01>$ \\
\hline Astigmatism $Z(2, \pm 2)(\mu \mathrm{m})$ & $2.21 \pm 1.49(0.05,9.38)$ & $0.89 \pm 0.74(0.01,3.71)$ & $0.01>$ \\
\hline Coma Z $(3, \pm 1)(\mu \mathrm{m})$ & $1.36 \pm 0.97(0.10,6.44)$ & $0.15 \pm 0.09(0.02,0.69)$ & $0.01>$ \\
\hline Spheric Z $(4,0)(\mu \mathrm{m})$ & $0.30 \pm 0.36(0.01,2.87)$ & $0.13 \pm 0.04(0.03,0.28)$ & $0.01>$ \\
\hline Residual HOAs $(\mu \mathrm{m})$ & $0.72 \pm 0.63(0.09,4.96)$ & $0.16 \pm 0.10(0.06,0.79)$ & $0.01>$ \\
\hline \multicolumn{4}{|l|}{ Posterior } \\
\hline $\mathrm{OPD}(\mu \mathrm{m})$ & $0.94 \pm 0.82(0.12,5.71)$ & $0.27 \pm 0.40(0.09,4.42)$ & $0.01>$ \\
\hline $\operatorname{HOAs}(\mu \mathrm{m})$ & $0.75 \pm 0.69(0.08,5.32)$ & $0.21 \pm 0.39(0.05,4.12)$ & $0.01>$ \\
\hline Astigmatism $Z(2, \pm 2)(\mu \mathrm{m})$ & $0.49 \pm 0.46(0.01,2.60)$ & $0.15 \pm 0.14(0.04,1.59)$ & $0.01>$ \\
\hline Coma Z $(3, \pm 1)(\mu)$ & $0.35 \pm 0.33(0.02,1.88)$ & $0.04 \pm 0.11(0,1.31)$ & $0.01>$ \\
\hline Spheric Z $(4,0)(\mu \mathrm{m})$ & $0.12 \pm 0.14(0,1.27)$ & $0.03 \pm 0.15(0,1.72)$ & $0.01>$ \\
\hline Residual HOAs $(\mu \mathrm{m})$ & $0.74 \pm 1.53(0.06,16)$ & $0.20 \pm 0.35(0.04,3.64)$ & $0.01>$ \\
\hline
\end{tabular}

* Mann-Whitney $U$ test. SD: standard deviation; $\mathrm{rf}=$ apical radius of the flattest meridian of the aspherotoric surface in $4.5 \mathrm{~mm}$ zone of the cornea; $\mathrm{rs}=$ apical radius of the steepest meridian of the aspherotoric surface in $4.5 \mathrm{~mm}$ zone of the cornea; $\mathrm{Q}=$ mean asphericity in $4.5 \mathrm{~mm}$ zone of the cornea; RMS/A: root mean square values per unit area in $4.5 \mathrm{~mm}$ zone of the cornea, $\mathrm{OPD}=$ total wavefront error; HOAs = higher-order aberrations.

TABLE 3: Receiver operating characteristic (ROC) analysis of the parameters where AUROC values are less than $0.995 \%$ confidence interval.

\begin{tabular}{|c|c|c|c|c|c|c|c|}
\hline Parameter & AUROC & SE & Lower & Upper & Cut-off value & Sensitivity (\%) & Specificity (\%) \\
\hline \multicolumn{8}{|l|}{ Summary indices } \\
\hline TCT $r(\mathrm{~mm})$ & 0.590 & 0.036 & 0.518 & 0.661 & 0.63 & 48.8 & 68.3 \\
\hline Apex $r(\mathrm{~mm})$ & 0.616 & 0.037 & 0.544 & 0.688 & 1.50 & 78.4 & 50 \\
\hline $\mathrm{AD}(\mathrm{mm})$ & 0.630 & 0.035 & 0.560 & 0.699 & 3.28 & 53.6 & 66.7 \\
\hline $\mathrm{CV}\left(\mathrm{mm}^{3}\right)$ & 0.821 & 0.027 & 0.769 & 0.874 & 56.25 & 77.6 & 73.3 \\
\hline \multicolumn{8}{|l|}{ Keratometry readings } \\
\hline Sim-K1 (D) & 0.746 & 0.031 & 0.685 & 0.807 & 43.93 & 53.6 & 86.7 \\
\hline Sim-K2 (D) & 0.873 & 0.022 & 0.829 & 0.917 & 45.32 & 79.2 & 81.7 \\
\hline Anterior $\varnothing=3 \mathrm{~mm} \mathrm{~K} 1$ (D) & 0.779 & 0.029 & 0.722 & 0.836 & 43.99 & 56.8 & 88.3 \\
\hline Anterior $\varnothing=5 \mathrm{~mm} \mathrm{K1}(\mathrm{D})$ & 0.748 & 0.031 & 0.687 & 0.810 & 44.05 & 53.6 & 89.2 \\
\hline Anterior $\varnothing=5 \mathrm{~mm} \mathrm{~K} 2(\mathrm{D})$ & 0.879 & 0.022 & 0.836 & 0.922 & 45.32 & 80 & 81.7 \\
\hline Posterior $\emptyset=3 \mathrm{~mm} \mathrm{K1}$ (D) & 0.713 & 0.036 & 0.642 & 0.785 & -6.23 & 59.2 & 95.8 \\
\hline Posterior $\emptyset=5 \mathrm{~mm} \mathrm{~K} 1$ (D) & 0.713 & 0.035 & 0.644 & 0.782 & -6.27 & 54.4 & 96.7 \\
\hline Posterior $\emptyset=5 \mathrm{~mm} \mathrm{~K} 2$ (D) & 0.886 & 0.023 & 0.840 & 0.932 & -6.58 & 82.4 & 85.8 \\
\hline \multicolumn{8}{|l|}{ Shape indices } \\
\hline Anterior rf (D) & 0.802 & 0.029 & 0.745 & 0.858 & 44.04 & 63.2 & 88.3 \\
\hline Anterior Q & 0.784 & 0.031 & 0.723 & 0.844 & 0.785 & 73.6 & 77.5 \\
\hline Posterior rf (D) & 0.864 & 0.026 & 0.814 & 0.914 & -6.31 & 71.2 & 98.3 \\
\hline Posterior Q & 0.796 & 0.031 & 0.736 & 0.856 & 0.495 & 72.8 & 83.3 \\
\hline \multicolumn{8}{|l|}{ Aberrations } \\
\hline \multicolumn{8}{|l|}{ Total } \\
\hline $\mathrm{OPD}(\mu \mathrm{m})$ & 0.871 & 0.024 & 0.825 & 0.917 & 1.30 & 86.4 & 81.7 \\
\hline Astigmatism $Z(2, \pm 2)(\mu \mathrm{m})$ & 0.808 & 0.029 & 0.752 & 0.864 & 1.10 & 76 & 79.2 \\
\hline Spheric $Z(4,0)(\mu \mathrm{m})$ & 0.575 & 0.039 & 0.498 & 0.652 & 0.205 & 39.2 & 90.8 \\
\hline \multicolumn{8}{|l|}{ Anterior } \\
\hline $\mathrm{OPD}(\mu \mathrm{m})$ & 0.881 & 0.022 & 0.837 & 0.925 & 1.475 & 85.6 & 85 \\
\hline Astigmatism Z $(2, \pm 2)(\mu \mathrm{m})$ & 0.809 & 0.028 & 0.755 & 0.864 & 1.285 & 72.8 & 80.8 \\
\hline Spheric $\mathrm{Z}(4,0)(\mu \mathrm{m})$ & 0.638 & 0.038 & 0.563 & 0.713 & 0.215 & 44 & 96.7 \\
\hline \multicolumn{8}{|l|}{ Posterior } \\
\hline Astigmatism $Z(2, \pm 2)(\mu \mathrm{m})$ & 0.842 & 0.028 & 0787 & 0.898 & 0.245 & 73.6 & 95 \\
\hline Spheric $Z(4,0)(\mu \mathrm{m})$ & 0.897 & 0.022 & 0.854 & 0.939 & 0.035 & 75.2 & 93.3 \\
\hline Residual HOAs $(\mu \mathrm{m})$ & 0.877 & 0.023 & 0.832 & 0.922 & 0.255 & 81.6 & 83.3 \\
\hline
\end{tabular}

AUROC: area under the ROC curve; SE: standard error; Apex $r=$ the distance of apex to the central cornea; $\mathrm{AD}=$ the depth of anterior chamber; CV $=$ corneal volume; Sim-K1 = simulated flat keratometry; Sim-K2 = simulated steep keratometry; K1 = flat keratometry; K2 = steep keratometry; $\mathrm{rf}=$ apical radius of the flattest meridian of the aspherotoric surface in $4.5 \mathrm{~mm}$ zone of the cornea; $\mathrm{Q}=$ mean asphericity in $4.5 \mathrm{~mm}$ zone of the cornea; OPD OPD = total wavefront error; HOAs = higher-order aberrations. 
TABLE 4: Receiver operating characteristic (ROC) analysis of the parameters where AUROC values are greater than 0.9 95\% confidence interval.

\begin{tabular}{|c|c|c|c|c|c|c|c|}
\hline Parameter & AUROC & SE & Lower & Upper & Cut-off value & Sensitivity (\%) & Specificity (\%) \\
\hline $\begin{array}{l}\text { Corneal indices } \\
\text { TCT }(\mu \mathrm{m}) \\
K_{\max }(\mathrm{D}) \\
\text { CCT }(\mu \mathrm{m})\end{array}$ & $\begin{array}{l}0.956 \\
0.927 \\
0.934 \\
\end{array}$ & $\begin{array}{l}0.012 \\
0.016 \\
0.015 \\
\end{array}$ & $\begin{array}{l}0.933 \\
0.895 \\
0.904 \\
\end{array}$ & $\begin{array}{l}0.979 \\
0.959 \\
0.963 \\
\end{array}$ & $\begin{array}{l}500.5 \\
47.85 \\
511.5 \\
\end{array}$ & $\begin{array}{c}90.4 \\
84 \\
87.2 \\
\end{array}$ & $\begin{array}{l}90.8 \\
88.3 \\
88.3 \\
\end{array}$ \\
\hline $\begin{array}{l}\text { Specially calculated indices } \\
\operatorname{Kmax} / \mathrm{TCT}(\mathrm{D} / \mu \mathrm{m}) \\
\mathrm{Kmax}^{2} / \mathrm{TCT}\left(\mathrm{D}^{2} / \mu \mathrm{m}\right)\end{array}$ & $\begin{array}{c}0.974 \\
0,975 \\
\end{array}$ & $\begin{array}{l}0.009 \\
0.008 \\
\end{array}$ & $\begin{array}{l}0.957 \\
0.959 \\
\end{array}$ & $\begin{array}{l}0.991 \\
0.991 \\
\end{array}$ & $\begin{array}{c}0.097 \\
0.0001 \\
\end{array}$ & $\begin{array}{l}89.6 \\
89.6 \\
\end{array}$ & $\begin{array}{l}97.5 \\
97.5 \\
\end{array}$ \\
\hline $\begin{array}{l}\text { Keratometry readings } \\
\text { Anterior } \emptyset=3 \mathrm{~mm} \mathrm{K2} \text { (D) } \\
\text { Posterior } \emptyset=3 \mathrm{~mm} \mathrm{K2} \text { (D) }\end{array}$ & $\begin{array}{l}0.901 \\
0.919 \\
\end{array}$ & $\begin{array}{c}0.020 \\
0.02 \\
\end{array}$ & $\begin{array}{l}0.862 \\
0.877 \\
\end{array}$ & $\begin{array}{l}0.940 \\
0.961 \\
\end{array}$ & $\begin{array}{l}45.60 \\
-6.73 \\
\end{array}$ & $\begin{array}{l}81.6 \\
85.6 \\
\end{array}$ & $\begin{array}{c}86.7 \\
95 \\
\end{array}$ \\
\hline $\begin{array}{l}\text { Shape indices } \\
\text { Anterior rs (D) } \\
\text { Anterior RMS/A }\left(\mu \mathrm{m} / \mathrm{mm}^{2}\right) \\
\text { Posterior rs (D) } \\
\text { Posterior RMS/A }\left(\mu \mathrm{m} / \mathrm{mm}^{2}\right)\end{array}$ & $\begin{array}{l}0.909 \\
0.965 \\
0.906 \\
0.974 \\
\end{array}$ & $\begin{array}{l}0.019 \\
0.013 \\
0.023 \\
0.011 \\
\end{array}$ & $\begin{array}{l}0.871 \\
0.940 \\
0.861 \\
0.953 \\
\end{array}$ & $\begin{array}{l}0.947 \\
0.990 \\
0.951 \\
0.995 \\
\end{array}$ & $\begin{array}{l}46.36 \\
0.065 \\
-6.70 \\
0.165 \\
\end{array}$ & $\begin{array}{c}79.2 \\
90.4 \\
84 \\
92.8 \\
\end{array}$ & $\begin{array}{l}94.2 \\
95.8 \\
95.8 \\
96.7\end{array}$ \\
\hline $\begin{array}{l}\text { Keratoconus indices } \\
\mathrm{SI}_{\mathrm{f}}(\mathrm{D}) \\
\mathrm{KV}_{\mathrm{f}}(\mu \mathrm{m}) \\
\mathrm{BCV}_{\mathrm{f}}(\mathrm{D}) \\
\mathrm{SI}_{\mathrm{b}}(\mathrm{D}) \\
\mathrm{KV}_{\mathrm{b}}(\mu \mathrm{m}) \\
\mathrm{BCV}_{\mathrm{b}}(\mathrm{D})\end{array}$ & $\begin{array}{l}0.950 \\
0.974 \\
0.976 \\
0.936 \\
0.965 \\
0.965\end{array}$ & $\begin{array}{c}0.017 \\
0.009 \\
0.01 \\
0.082 \\
0.013 \\
0.013\end{array}$ & $\begin{array}{l}0.917 \\
0.956 \\
0.956 \\
0.897 \\
0.939 \\
0.940\end{array}$ & $\begin{array}{l}0.983 \\
0.992 \\
0.996 \\
0.974 \\
0.990 \\
0.990\end{array}$ & $\begin{array}{c}0.89 \\
9.5 \\
0.51 \\
0.225 \\
20.5 \\
0.555\end{array}$ & $\begin{array}{c}88.8 \\
92 \\
92.8 \\
88.8 \\
92 \\
88.8\end{array}$ & $\begin{array}{l}98.3 \\
94.2 \\
96.7 \\
98.3 \\
95.8 \\
97.5\end{array}$ \\
\hline $\begin{array}{l}\text { Aberrations } \\
\text { Total } \\
\text { HOAs }(\mu \mathrm{m}) \\
\text { Coma } Z(3, \pm 1)(\mu \mathrm{m}) \\
\text { Residual }(\mu \mathrm{m})\end{array}$ & $\begin{array}{l}0.940 \\
0.933 \\
0.907\end{array}$ & $\begin{array}{l}0.016 \\
0.017 \\
0.020\end{array}$ & $\begin{array}{l}0.909 \\
0.899 \\
0.868\end{array}$ & $\begin{array}{l}0.972 \\
0.966 \\
0.945\end{array}$ & $\begin{array}{c}0.60 \\
0.39 \\
0.295\end{array}$ & $\begin{array}{l}85.6 \\
81.6 \\
90.4\end{array}$ & $\begin{array}{l}93.3 \\
96.7 \\
79.2\end{array}$ \\
\hline $\begin{array}{l}\text { Anterior } \\
\text { HOAs }(\mu \mathrm{m}) \\
\text { Coma } Z(3, \pm 1)(\mu \mathrm{m}) \\
\text { Residual }(\mu \mathrm{m}) \\
\end{array}$ & $\begin{array}{l}0.975 \\
0.963 \\
0.950 \\
\end{array}$ & $\begin{array}{l}0.009 \\
0.011 \\
0.014 \\
\end{array}$ & $\begin{array}{l}0.958 \\
0.941 \\
0.923 \\
\end{array}$ & $\begin{array}{l}0.992 \\
0.986 \\
0.977 \\
\end{array}$ & $\begin{array}{l}0.575 \\
0.375 \\
0.270 \\
\end{array}$ & $\begin{array}{l}88.8 \\
87.2 \\
89.6 \\
\end{array}$ & $\begin{array}{l}97.5 \\
97.5 \\
89.2 \\
\end{array}$ \\
\hline $\begin{array}{l}\text { Posterior } \\
\text { OPD }(\mu \mathrm{m}) \\
\operatorname{HOAs}(\mu \mathrm{m}) \\
\text { Coma } Z(3, \pm 1)(\mu \mathrm{m})\end{array}$ & $\begin{array}{l}0.911 \\
0.913 \\
0.959\end{array}$ & $\begin{array}{l}0.020 \\
0.019 \\
0.014\end{array}$ & $\begin{array}{l}0.870 \\
0.876 \\
0.933\end{array}$ & $\begin{array}{l}0.951 \\
0.951 \\
0.986\end{array}$ & $\begin{array}{l}0.375 \\
0.305 \\
0.075\end{array}$ & $\begin{array}{c}85.6 \\
84 \\
91.2\end{array}$ & $\begin{array}{c}90 \\
89.2 \\
97.5\end{array}$ \\
\hline
\end{tabular}

AUROC: area under the ROC curve; SE: standard error; TCT = minimum corneal thickness; $\mathrm{K}_{\max }=$ keratometry of the steepest point recorded from the anterior tangential map; CCT = central corneal thickness; K2 = steep keratometry; rs = apical radius of the steepest meridian of the aspherotoric surface in $4.5 \mathrm{~mm}$ zone of the cornea; $\mathrm{RMS} / \mathrm{A}$ : root mean square values per unit area in $4.5 \mathrm{~mm}$ zone of the cornea; $\mathrm{SIf}=$ symmetry index front; $\mathrm{KVf}=\mathrm{keratoconus} \mathrm{vertex}$ front index; $\mathrm{BCVf}=$ Baiocchi Calossi Versaci front index; $\mathrm{SIb}=$ symmetry index back; $\mathrm{KVb}=$ keratoconus vertex back index; $\mathrm{BCVb}=\mathrm{Baiocchi} \mathrm{Calossi} \mathrm{Versaci}$ back index; $\mathrm{OPD}=$ total wavefront error; $\mathrm{HOAs}=$ higher-order aberrations $\mathrm{OPD}=$ total wavefront error; $\mathrm{HOAs}=$ higher-order aberrations.

the AUROC values of the summary indices and $\mathrm{K}$ readings. $\mathrm{BCV}_{\mathrm{f}}$ had the highest AUROC value (0.976) in the study.

The LRA models built for differentiating keratoconus eyes from normal eyes are in Table 5. The most successful LRA was defined in Model 8 with an accuracy of $97.5 \%$, sensitivity of $96.8 \%$, and specificity of $99.2 \%$ ( -2 log-likelihood score: 42.461). It is formulated as follows: $\operatorname{logit}=3,471+\left(5,431 \mathrm{x} \quad \mathrm{BCV}_{\mathrm{f}}\right)+\left(6,533 \mathrm{x} \quad \mathrm{BCV}_{\mathrm{b}}\right)+(-1.883 \mathrm{x}$ posterior rf $)+(-0,031 x$ CCT $)+(-0,007 x$ TCT $)$. In Model 8 , the significance levels were $\mathrm{BCV}_{\mathrm{f}}(p=0.001), \mathrm{BCV}_{\mathrm{b}}$ $(p=0.002)$, posterior $\mathrm{rf}(p=0.005)$, CCT $(p=0.072)$, and TCT $(p=0.494)$. The AUROC and cut-off values of the logistic function formula of Model 8 were 0.992 and -0,0225, respectively.

\section{Discussion}

Many technologies have been developed to distinguish keratoconus eyes from normal eyes. The efficacy of Scheimpflug-Placido imaging in differentiating keratoconus eyes from normal eyes has been investigated in previous studies [9].

LRA was used to determine the most predictive model for keratoconus detection in this study, and our LRA model (model 8) can differentiate keratoconus eyes from normal eyes with high accuracy (97.5\%), sensitivity (96.8\%), and specificity (99.2\%). Ucakhan et al. [10] reported a similar performance with their logistic regression model (sensitivity 97.7\% and specificity 95.2\%) with 11 topographic 
TABLE 5: Performance of the logistic regression analysis models for differentiating keratoconus eyes from normal eyes.

\begin{tabular}{lccc}
\hline Model & Sensitivity & Specificity & -2 log-likelihood \\
\hline Model 1 & $\% 91.2$ & $\% 95.8$ & 94,624 \\
Model 2 & $\% 89,6$ & $\% 97,5$ & 98,971 \\
Model 3 & $\% 91,2$ & $\% 98,3$ & 84,663 \\
Model 4 & $\% 91,2$ & $\% 97,5$ & 79,305 \\
Model 5 & $\% 93,6$ & $\% 98,3$ & 59,774 \\
Model 6 & $\% 94,4$ & $\% 98,3$ & 44,416 \\
Model 7 & $\% 96,8$ & $\% 99.2$ & 47,993 \\
Model 8 & $\% 96,8$ & $\% 99.2$ & 42,461 \\
\hline
\end{tabular}

$\mathrm{K} 2+\mathrm{K}_{\max }+\mathrm{CCT}+\mathrm{TCT} ;$ Model $2=$ anterior $\mathrm{rf}+$ anterior $\mathrm{rs}+$ anterior $\mathrm{Q}+$ anterior RMS/A; Model $3=$ posterior $\mathrm{rf}+$ posterior $\mathrm{rs}+$ posterior $\mathrm{Q}+$ posterior RMS/A; Model 4= Model 2+ Model 3; Model $5=\mathrm{BCV}_{\mathrm{f}}+\mathrm{BCV}_{\mathrm{b}}+\mathrm{KV}_{\mathrm{f}}+\mathrm{KV}_{\mathrm{b}}+\mathrm{SI}_{\mathrm{f}}+\mathrm{SI}_{\mathrm{b}}$; Model $6=$ all of the aberrations on Table 2; Model $7=\mathrm{BCV}_{\mathrm{f}}+\mathrm{BCV}_{\mathrm{b}}+$ posterior $\mathrm{rf}+\mathrm{KVf}+$ anterior coma $\mathrm{Z}$ $(3, \pm 1)$; Model $8=\mathrm{BCV}_{\mathrm{f}}+\mathrm{BCV}_{\mathrm{b}}+$ posterior $\mathrm{rf}+\mathrm{TCT}+\mathrm{CCT}$.

parameters, while our LRA model can perform similarly with only five parameters. We believe that using five parameters will save time for ophthalmologists. The logistic function formula in our study can be used with an Excel file (Microsoft Office, Microsoft Corp., Redmond, WA, USA). Results greater than the cut-off value $(-0,1512)$ will indicate keratoconus diagnosis.

There are keratoconus detection algorithms and computer-aided diagnosis systems based on the data of Scheimpflug corneal topography devices in the literature. These have a discriminating ability that is higher than our regression model with an accuracy of $97.2 \%-99.5 \%$ [11-14] and sensitivity of $99.1 \%-100 \%$. [11, 15], but the LRA model in our study can diagnose keratoconus without the need for complex computer algorithms and software.

The $\mathrm{BCV}_{\mathrm{f}}$ was found to be the most significant parameter in the logistic regression model $(p=0.001)$. It is obtained by a linear combination of vertical trefoil $Z(3,-3)$, vertical coma $Z(3,-1)$, horizontal coma $Z(3,+1)$, primary spherical aberration $Z(4,0)$, and second-order vertical coma $Z(5,-1)$ and by processing them with a function of the coma axis on the anterior Zernike decomposition. [11].

This study is the most extensive study examining the AUROC values of the Sirius Scheimpflug-Placido system's parameters. ROC analysis of 51 parameters was evaluated and graded them according to their AUROC value. TCT, $\mathrm{BCV}_{\mathrm{f}}, \mathrm{KV}_{\mathrm{f}}$, anterior RMS/A, $\mathrm{BCV}_{\mathrm{b}}, \mathrm{KV}_{\mathrm{b}}$, posterior RMS/A, $\mathrm{Kmax}^{2} / \mathrm{TCT}$, anterior HOAs, anterior coma, and posterior coma were found to be the most valuable parameters for differentiating keratoconus eyes from normal eyes.

In the literature concerning the diagnosis of keratoconus, there is some controversy in the ROC analysis of topographic parameters. Due to variations in the settings of the topography systems and study populations, cut-off values may be different from each other in various studies.

Toprak et al. [16] reported the AUROC value of $\mathrm{Kmax}^{2}$ / TCT as 0.997 (sensitivity $>99 \%$, specificity $>94 \%$ ). In our study, although the AUROC value (0.975) and sensitivity (89.6\%) were not as high as those in Toprak et al. [16], the AUROC value of $\mathrm{Kmax}^{2} / \mathrm{TCT}$ was very close to the AUROC value of BCVf (0.976), which had the highest value in our study. We believe that $\mathrm{Kmax}^{2} / \mathrm{TCT}$ is a valuable parameter for discriminating keratoconus eyes from normal eyes.

RMS/A defines root mean square values per unit area in the $4.5 \mathrm{~mm}$ zone of the cornea and posterior RMS/A was found to be one of the most significant AUROC values (0.974) in the study. Shetty et al. reported AUROC values of 0.954 for anterior RMS/A and 0.983 for posterior RMS/A. [17] Their results are similar to ours, but their cut-off values (anterior $\mathrm{RMS} / \mathrm{A}=0.131 \mu \mathrm{m} / \mathrm{mm}^{2}$ and posterior $\mathrm{RMS} / \mathrm{A}=0.269 \mu \mathrm{m} /$ $\mathrm{mm}^{2}$ ) are higher than our values (anterior RMS/ $\mathrm{A}=0.065 \mu \mathrm{m} / \mathrm{mm}^{2}$ and posterior RMS $/ \mathrm{A}=0.165 \mu \mathrm{m} / \mathrm{mm}^{2}$ ).

Shetty et al. [17] also reported that the AUROC values of posterior keratoconus indices $\left(\mathrm{SI}_{\mathrm{b}}=0.941 ; \mathrm{BCV}_{\mathrm{b}}=0.969\right)$ are higher than the anterior keratoconus indices $\left(\mathrm{SI}_{\mathrm{f}}=0,921\right.$; $\mathrm{BCV}_{\mathrm{f}}=0.940$ ), but we found the opposite. In our study, the AUROC values of the anterior keratoconus indices $\left(\mathrm{SI}_{\mathrm{f}}=0.950 ; \mathrm{KV}_{\mathrm{f}}=0.974 ; \mathrm{BCV}_{\mathrm{f}}=0.976\right)$ were higher than the AUROC values of the posterior keratoconus indices $\left(\mathrm{SI}_{\mathrm{b}}=0.936 ; \mathrm{KV}_{\mathrm{b}}=0.965 ; \mathrm{BCV}_{\mathrm{b}}=0.965\right)$, and $\mathrm{BCV}_{\mathrm{f}}$ had the highest AUROC value.

The fact that both eyes of the patients were included in the study is one of the limitations of the study. The measurements from both eyes of the same subject tend to be positively correlated, and including only one eye per individual will give more accurate results [18]. Another limitation of the study is that keratoconus patients were not categorized in mild, moderate, and advanced cases, and the ability of the LRA model to detect subclinical keratoconus cases has not been investigated.

In conclusion, the LRA model in the study can distinguish keratoconus corneas from normal ones with high accuracy without the need for complex computer algorithms and software, but the study population is relatively small. Further studies with more patients are needed to reveal the efficacy of our results to diagnose keratoconus.

\section{Data Availability}

The logistic function formula in our study can be used with an Excel file (Microsoft Office, Microsoft Corp., Redmond, WA, USA). The data used to support the findings of this study and the logistic regression formula in an Excel file are available from the corresponding author upon request.

\section{Disclosure}

No financial support was received for this submission.

\section{Conflicts of Interest}

None of the authors have any conflicts of interest.

\section{Acknowledgments}

The study was presented as an oral presentation at the 53rd National Congress of the Turkish Ophthalmological Association on 7 November 2019 in Antalya, Turkey. 


\section{References}

[1] J. C. Reddy, C. J. Rapuano, J. R. Cater, K. Suri, P. K. Nagra, and K. M. Hammersmith, "Comparative evaluation of dual scheimpflug imaging parameters in keratoconus, early keratoconus, and normal eyes," Journal of Cataract and Refractive Surgery, vol. 40, no. 4, pp. 582-592, 2014.

[2] P. S. Binder, "Ectasia after laser in situ keratomileusis," Journal of Cataract and Refractive Surgery, vol. 29, no. 12, pp. 24192429, 2003.

[3] D. Varssano, I. Kaiserman, and R. Hazarbassanov, "Topographic patterns in refractive surgery candidates," Cornea, vol. 23, no. 6, pp. 602-607, 2004.

[4] J. B. Randleman, B. Russell, M. A. Ward, K. P. Thompson, and R. D. Stulting, "Risk factors and prognosis for corneal ectasia after LASIK," Ophthalmology, vol. 110, no. 2, pp. 267-275, 2003.

[5] O. Muftuoglu, O. Ayar, K. Ozulken, E. Ozyol, and A. Akıncı, "Posterior corneal elevation and back difference corneal elevation in diagnosing forme fruste keratoconus in the fellow eyes of unilateral keratoconus patients," Journal of Cataract and Refractive Surgery, vol. 39, no. 9, pp. 1348-1357, 2013.

[6] D. P. Piñero, J. L. Alió, A. Alesón, M. E. Vergara, and M. Miranda, "Corneal volume, pachymetry, and correlation of anterior and posterior corneal shape in subclinical and different stages of clinical keratoconus," Journal of Cataract and Refractive Surgery, vol. 36, no. 5, pp. 814-825, 2010.

[7] Y. G. D. Genc, E. Tuccar, and B. Yagmurlu, "Estimation of sensitivity and specificity for clustered data," Turkish Journal of Medical Sciences, vol. 35, pp. 21-24, 2005.

[8] G. A. Diamond, "What price perfection? calibration and discrimination of clinical prediction models," Journal of Clinical Epidemiology, vol. 45, no. 1, pp. 85-89, 1992.

[9] R. Montalbán, J. L. Alió, J. Javaloy, and D. P. Piñero, "Intrasubject repeatability in keratoconus-eye measurements obtained with a new scheimpflug photography-based system," Journal of Cataract and Refractive Surgery, vol. 39, no. 2, pp. 211-218, 2013.

[10] Ö. Ö. Uçakhan, V. Çetinkor, M. Özkan, and A. Kanpolat, "Evaluation of scheimpflug imaging parameters in subclinical keratoconus, keratoconus, and normal eyes," Journal of Cataract and Refractive Surgery, vol. 37, no. 6, pp. 1116-1124, 2011.

[11] M. C. Arbelaez, F. Versaci, G. Vestri, P. Barboni, and G. Savini, "Use of a support vector machine for keratoconus and subclinical keratoconus detection by topographic and tomographic data," Ophthalmology, vol. 119, no. 11, pp. 2231-2238, 2012.

[12] I. Ruiz Hidalgo, P. Rodriguez, J. J. Rozema et al., "Evaluation of a machine-learning classifier for keratoconus detection based on scheimpflug tomography," Cornea, vol. 35, no. 6, pp. 827-832, 2016.

[13] A. Lavric and P. Valentin, "Kerato. Detect: keratoconus detection algorithm using convolutional neural networks," Computational Intelligence and Neuroscience, vol. 2019, Article ID 8162567, 9 pages, 2019.

[14] I. Issarti, A. Consejo, M. Jiménez-García, S. Hershko, C. Koppen, and J. J. Rozema, "Computer aided diagnosis for suspect keratoconus detection," Computers in Biology and Medicine, vol. 109, pp. 33-42, 2019.

[15] D. Smadja, D. Touboul, A. Cohen et al., "Detection of subclinical keratoconus using an automated decision tree classification," American Journal of Ophthalmology, vol. 156, no. 2, pp. 237-246, 2013.
[16] I. Toprak, V. Yaylalı, and C. Yildirim, "A combination of topographic and pachymetric parameters in keratoconus diagnosis," Contact Lens and Anterior Eye, vol. 38, no. 5, pp. 357-362, 2015.

[17] R. Shetty, H. Rao, P. Khamar et al., "Keratoconus screening indices and their diagnostic ability to distinguish normal from ectatic corneas," American Journal of Ophthalmology, vol. 181, pp. 140-148, 2017.

[18] H. G. Zhang and G.-S. Ying, "Statistical approaches in published ophthalmic clinical science papers: a comparison to statistical practice two decades ago," British Journal of Ophthalmology, vol. 102, no. 9, pp. 1188-1191, 2018. 